\title{
Remanufacturing in automotive industry: Challenges and limitations
}

\author{
Paulina Golinska ${ }^{1}$, Arkadiusz Kawa ${ }^{2}$ \\ ${ }^{1}$ Poznan University of Technology, Strzelecka 11, 60-965 Poznan, (POLAND) \\ ${ }^{2}$ Poznan University of Economics, al. Niepodlegtości 10, 61-875 Poznan, (POLAND) \\ paulina.golinska@put.poznan.pl; arkadiusz.kawa@ue.poznan.pl
}

Received June 2010

Accepted September 2011

\section{Abstract:}

Purpose: The aim of this paper is to provide the framework for management of reverse flow of materials in automotive industry. The emphasis is placed on the remanufacturing activities. Materials management in such conditions is a real challenge. The cause for this is parallel use of raw materials and reused materials. Such hybrid flows of materials are characterized by increased level of uncertainty connected with amount, quality and timing.

Design/methodology/approach: This paper presents a comprehensive review of remanufacturing and traditional manufacturing. The stabilization of reverse flows is crucial for continuity of remanufacturing operations. The simulation model and results are discussed regarding stabilization of the reverse flows.

Findings: Authors identify main problems that appear in the area of combining at the production system forward and reverse flows of materials. The agent-based technology is applied for configuration and stabilization of reverse network.

Research limitations/implications: Paper is case - oriented.

Practical implications: Both logistician and IT researchers might benefit from authors approach. 
Originality/value: Authors provide an interdisciplinary approach combining operations management, logistics and information technology.

Keywords: reverse logistics, remanufacturing, closed loop supply chain, simulation experiments

\section{Introduction}

Automotive industry is one of the most environmental aware manufacturing sectors. The shift in business practice from traditional manufacturing to eco-friendly solutions was caused mainly by the application of a number of European Union Directives (e.g. End-of-Life Vehicles ELV Directive 2000/53/EC or RRR 2005/64/EC). Also Extended Producer Responsibility Policy must be fulfilled. Product take-back regulations influence design of the vehicles, production technologies but also the configuration of automotive supply chains. The business practice is every year closer to the closed loop supply chain concept. The ideal closed loop supply chain can be defined as zero-waste supply chain that completely reuses, recycles, or composts all materials (Golinska et al., 2007). All materials originally used in manufacturing of product should be preferable reused at the end of life in the forward supply chain, as so called re-supply. In this paper goal of closed loop chain materials management is defined as a need to provide the continuity of remanufacturing activities and simultaneously to minimize the number of waste being generated the in whole supply chain. The aim of this contribution is to provide the framework for management of reverse flow of materials in automotive industry. The emphasis is placed on the remanufacturing perspective.

\section{Closed loop supply chain and remanufacturing fundamentals}

In last decade closed-loop supply chain (CLSC) concept has been widely discussed in the literature on logistics and operation management. General overviews of closed loop supply chain concept can be found in (Guide \& van Wassenhove, 2003, Dekker et al., 2003). The forward supply chain processes includes all procurement and deliveries of raw materials, work-in-progress materials flow at production plant and distribution of products to the customers. The reverse processes in supply chain are more difficult to define. The appearance of particular activities within the CLSC depends very often on the condition/quality of the returns and source of collection. Main phases in reverse supply chain are collection, inspection and 
disassembly, reprocessing (recycling, remanufacturing or reuse) and re-supply or redistribution to alternative markets. In this paper the redistribution issues are not discussed.

Uncertainty is the main problem in materials management of combined forward and reverse flows. The uncertainty is caused by "mismatch between supply and demand with respect to timing and quantity in a recovery networks" (Fleischmann et al., 2001).

The materials management for remanufacturing purpose is determined by the closed loop supply chain type. In situation when manufacturer does not perceive the reverse flow as an area with cost-cutting potential then he does not make much effort in order to optimize reverse logistics operations.

In Europe the manufactures are mainly closing the supply loop due to the legislative requirements. European firms seem to rely on the waste stream for acquiring recoverable products (Guide, 2003). Recently automotive manufactures have started to apply less passive approach.

Remanufacturing can be defined as an industrial process in which new product is reassembly from an old one and, where necessary, new parts are used to produce fully equivalent and sometimes superior-in performance and expected lifetime to original new product (Lund, 1983). Remanufacturing is distinctly different from repair operations, since products are disassembled completely and all parts are returned to like-new conditions, which may include cosmetic operations (Guide, 2000).

Remanufacturing allows companies to capture the residual value-added in forms of materials, energy and labor. Remanufacturing profitability can driven the reverse flow of materials and makes companies to optimize their reverse channels. The remanufacturing operations can be divided into three phases:

- disassembly

- reprocessing of materials

- reassembly

Figure 1 represents the area of materials management in the closed loop supply chain (grey area). 


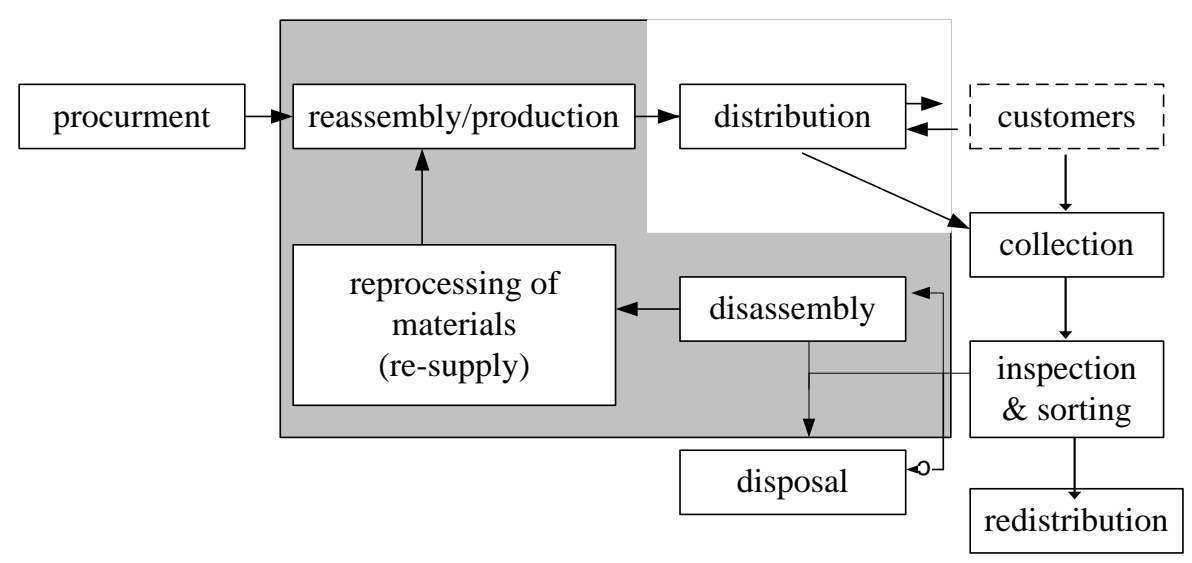

Figure 1. Closed loop supply chain model with remanufacturing

In order to identify the requirements for efficient materials management for remanufacturing purpose there is a need to identify the differences between traditional manufacturing and remanufacturing. Authors of the previous studies on remanufacturing have taken in consideration mainly following aspects of materials management:

- inventory control and management: inventory control models (van der Laan et al., 2003), ordering policy (Toktay, 2000)

- reverse network design and collection strategies (Fleischmann et al., 2000)

- $\quad$ matching demand and supply (Guide, 2003)

- material requirements planning (Ferrer \& Whybark, 2001)

There is a research gap regarding more complex approach to materials management and integration of materials and accompanying information flows.

\section{Industry insights - automotive remanufacturing}

The automotive industry is the highly consolidated sector, with the global overcapacity of about $30 \%$. The market is rather saturated and demand is driven by product replacement. Profit margin on new cars is relatively low, particularly in the low price/high volume segments of the market. The global downturn in sales in years 2008-2009 has forced automobile makers to review their structures and processes.

Moreover since 2002 EU has introduced End of Life Vehicle Directive that requires manufacturers to reach the goal of new vehicles reusability and/or recyclability of 
at least $85 \%$, and reusability and/or recoverability of at least $95 \%$ by weight, if measured against the international standard ISO 22620 (EAIR, 2009).

The RRR Directive 2005/64/EC on type approval of vehicles for reusability, recyclability, and recoverability came into force in December 2005 and requires cars and light vans (M1/N1), newly introduced to the market after December 2008 to be $85 \%$ reusable and/or recyclable and $95 \%$ reusable/recoverable by mass (SMMT, 2007). Focus on recyclability has driven the new model planning process. Newly applied advanced recycling methods (post shredder treatment) allow nowadays the recycling and recovery of literally all materials. Moreover there is a shift in design approach so called product modularity. It allows improving disassembly operations. To speed up the dismantling operations all components are labeled in accordance with international ISO standards, enabling materials to be sorted according to their type. In order to reach the challenging goal of $95 \%$ recovery target by 2015 some efficient material separation technologies for end-oflife vehicles are promoted that allow the utilization for shredder residue and boosting the usage of recycled materials for some specific car components.

The EU is the still the largest automotive production region $(27 \%)$ in the world, despite incremental growth of car production in China in 2010. At the end of 2008 in Europe there was altogether over 226 million of passenger's car in use, including 73 millions older than 10 years. The average age of car in "old" UE 16 countries was 8.2 years, including the rest of European countries it is increased to over 11 years (ACEA, 2010). The problem of old car is growing but this aftermarket trends also create opportunities, including a growing market for remanufactured products. Several characteristics of cars make this a difficult market in which to implement product recovery: a long and unpredictable working life, an ability to cross international boundaries, reselling between different owners, and, therefore, difficulties in tracking the product during its active life (Seitz \& Peattie, 2004). Remanufacturing for major car components, such as engines, is less problematic.

The automobile industry has the longest tradition in remanufacturing among all industries. The automotive products' remanufacturing accounts for two thirds of all remanufacturing activities globally (Kim et al., 2008). Remanufacturing is a common practice in automotive industry because $10 \%$ of all cars and trucks require an engine replacement during their life. Starters and alternators are, beside car engines the most typical products to be remanufactured due to the fact that most car require two of each throughout their lives. These components are mass produced and remanufactured by thousands of companies. About 300 starter motor 
and alternator remanufacturers are active worldwide. $50 \%$ of the companies are US producers; the other $30 \%$ of the companies are located in Europe. Their production volumes together are about 50 million pieces, which constitutes more than $80 \%$ of the overall remanufactured products (Kim et al., 2008).

In Europe, OEMs have just discovered the aftermarket profit potential of remanufactured products. There are three major market sectors: OEMs, including vehicle manufacturers and their first tier suppliers; remanufacturers subcontracted to the OEMs; and independent remanufacturers (Seitz \& Peattie, 2004). In this paper the emphasis is placed on OEMs, including vehicle manufacturers and their first tier suppliers.

\section{Remanufacturing vs. manufacturing}

Remanufacturing differs a lot from traditional manufacturing. Many authors have pointed as a key complication the need to deliver products from many locations to one processor in a "many to one transportation" or "many to one distribution points" system (Tibben-Lembke \& Rogers, 2002). Constructing the reverse channels is perceived as a major obstacle within closed loop supply chain management in terms of the physical locations, facilities, and transportation links that need to be established and managed (Fleischmann et al., 2001). In this paper authors highlight the problems that appear within OEM's facility.

In table 1 authors have presented the comparison of traditional manufacturing process and remanufacturing process in automotive industry.

\begin{tabular}{|l|l|l|}
\hline & Manufacturing & Remanufacturing \\
\hline Production Size & big/medium & medium/small \\
\hline $\begin{array}{l}\text { Product } \\
\text { Standardization }\end{array}$ & high/medium & medium/low \\
\hline $\begin{array}{l}\text { Materials and work - in- } \\
\text { progress inventory level }\end{array}$ & $\begin{array}{l}\text { just-in-time, just-in-sequence, } \\
\text { low safety buffers }\end{array}$ & $\begin{array}{l}\text { high - buffering against } \\
\text { uncertainty }\end{array}$ \\
\hline Inventory cycle & short & long \\
\hline $\begin{array}{l}\text { Inventory } \\
\text { replenishment }\end{array}$ & standardized procedures & ad hoc \\
\hline Process lead time & predictable & $\begin{array}{l}\text { variable/ not following } \\
\text { simple stochastic patterns }\end{array}$ \\
\hline Delivery lead time & predictable & Variable \\
\hline
\end{tabular}

Table 1. Manufacturing vs. Remanufacturing

By remanufacturing product standardization is much lower than by traditional production. Moreover the complication is caused by multiple products variants e.g. engines. Model explosion in customized mass production of new products seriously influence to possibility of batch optimization in remanufacturing area. The 
manufacturer only has to deal with one generation of product's variants at a time, and mass production volumes allow production lines to be dedicated to very limited products' models. The remanufacturer has to deal with small batches encompassing a range of product variants and generations, which complicates tool-changing, disassembly, and assembly processes (Seitz \& Peattie, 2004).

Most of the automotive manufacturers use advanced supplies methods like just-intime or just-in-sequence, which allow big reduction of the inventories levels. These approaches are not suitable for remanufacturing because of the uncertainty inherent in delivery lead times. Also products' proliferation influences the inventory levels at remanufacturing activities. The material recovery rate (MRR) is less predictable for the collected end-of-life vehicles. It results in higher level of workin-progress inventory (WIP) kept. MRR defines the frequency that a material recover from disassembled unit is suitable for further use in production activities.

Due to the variable MRR the returned products or raw materials to substitute not remanufacturable elements are stored longer than in traditional manufacturing (inventory cycle). A big problem is forecasting of the reverse material flows. It is difficult to use standardized approaches for used materials/parts inventory replenishment. Very often the inventory is replenished when lack of particular part/product type appears.

The remanufacturing process lead times differs significantly and depends on reprocessing activities needed in order to disassembly and recover the used parts. In general disassembly operations are highly variable with respect of time required. Moreover the reprocessing may include different operations for the same type of returns depending on their conditions. Some operations/tasks are known with certainty but appearance of others might be probabilistic. It makes setting accurate lead times for remanufacturing very difficult.

The main problems in materials management for remanufacturing purpose can be defined as:

- the uncertain timing and quantity of returns

- the uncertainty in materials recovered from return items

- the reverse logistics network configuration

- the problems of stochastic routings for materials for remanufacturing operations and highly variable processing times 
Most of the above mentioned characteristics result from lack of appropriate information on material flows and its forecasting. In the next section we present the simulation model to solve this problem.

\section{Simulation of materials management in automotive industry}

\subsection{Assumptions}

The closed loop supply chain model (CLSC) was implemented in a programmable modeling platform for simulating - NetLogo. It allows giving instructions to a plethora of independent agents interacting with one another and performing multiple tasks (Wilensky, 2009). In the simulation model three tiers have been distinguished. The first tier is represented by OEMs followed by recyclers and dealers. The OEM provides requirements for recovery. It collects and consolidates customers' individual return notices at specified time intervals (e.g. once a day) and passes them on to the right members of the configured supply chain. It has access to information about MRR, delivery time, etc., and supply (production capacity) offered by all enterprises from the network.

In the CLSC model goods and information flows take place between consecutive tiers. Among the subsequent tiers a flow of goods and information about them takes place. All goods deliveries are carried out sequentially from the supplier of the last tier (dealers) to the one of the first tier. Such an enterprise network comes in the form of a stratified, directed graph consisting of $n$ sources (dealers) and one sink (OEM).

In such a network the fastest flow (the shortest time) with an appropriate capacity is found. This task can be solved by the Busacker-Gowen algorithm, which is presented in (Deo et al., 1983). This method consists in increasing the flow along consecutive paths augmenting as much as their capacity allows. The order of appointing paths depends on their length which, in this case, is determined by unit time (disassembly, reprocessing, reassembly, and delivery time). If the flow achieves the defined value, computing finishes. Otherwise, the network is modified and the next stages are repeated until the flow of the predefined value is accomplished. To find the shortest chain from the source to the sink the algorithm of finding the shortest paths must be applied. The CLSC model has used the BMEP algorithm (Kawa, 2009). The quantity of the potential entities in each tier (except for the first tier) is assumed to range from 10 to 100. This number can be increased or decreased with a slider. There is only one OEM in each network (Figure 2). 
Different dealers from other reverse networks may compete with others (Kawa \& Golinska, 2010).

Two other parameters of this network have been distinguished: demand and indicator. The first one is the demand of the OEM, which equals the whole supply chain demand by day. The second one is a factor of the supply changeability of particular entities of the network.

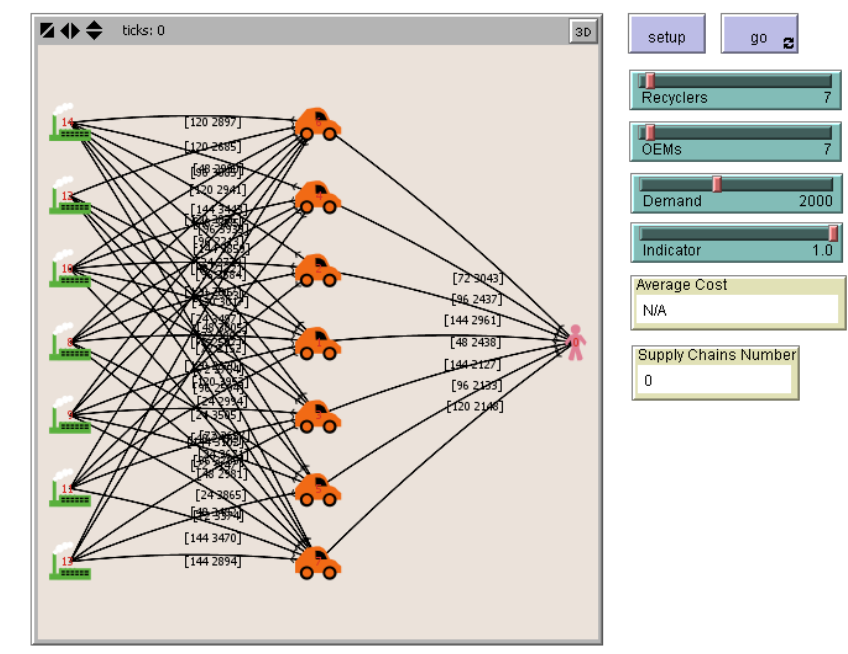

Figure 2. Reverse network of automotive industry in the NetLogo platform

The properties of link agents between constituents have been chosen randomly as a pair of time and capacity. We have set the time as a variable of $24,48,72,96$, 120 , or 144 hours. In turn, capacity is a variable which depends on the aforementioned indicator. The total of the flows outgoing from a given supplier to their recipients equals the supply value (production capacity) of the supplier in question. Because the OEM demand can be completely or partially satisfied, there can be one or more such chains.

Due to the fact that MRR is an important parameter, it has been assumed that in a given network there are only such connections between the suppliers of consecutive tiers which equal at least $50 \%$.

\subsection{Simulation experiments}

Checking the capabilities of reverse supply chain configuration and the effectiveness of the CLSC model has been carried out by means of numerous simulation experiments. Their aim has been to study how the changes of the nodes number and indicator influence the average supply chain numbers (avg-sc number) 
and the average time of the whole process of the recovery in the network (avgtime).

Indicator has been assumed to equal 0.1 and demand has been presumed to be 2000 for the first set of the simulation experiments. The number of entities in a particular tier has been changing and consecutively amounted to: $10,20,30, \ldots$, 100 (at the same time, this number in other tiers was stable and equaled 10). The simulations were run 1000 times for each case. The findings of the experiment show that as nodes number increases (from 10 to 100), avg-time declines by about $51 \%$ (from 99 to 48 hours) for recyclers and by 6\% for dealers (from 100 to 94 hours) (Figure 3 ).

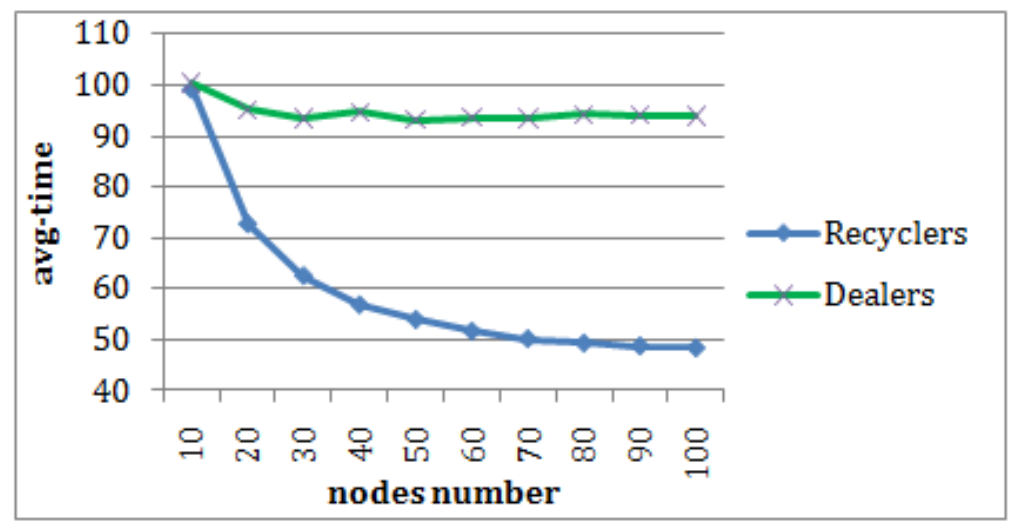

Figure 3. Influence of nodes number change in tiers on the average time of the whole process in the recovery network

The following dependency may provide a possible explanation for this phenomenon: the more suppliers there are in a given tier, the greater the competitiveness among them is and the shorter the time becomes for the final customers. Figure 2 also shows that the time decreases simultaneously with an increase in the nodes number in the vicinity of the OEM (i.e. recyclers).

In the next part of the simulations, the indicator variable has been shifted from 0.1 to 1.0 incrementing each time by 0.1 , on the assumption that nodes number is stable and amounts to 10 and demand $=2000$. The simulations were conducted 1000 times for each case. The results from the experiment show that avg-sc number falls from 10 to 1 (Figure 4). The fact that augmenting indicator twice (i.e. from 0.1 to 0.2 ) leads the average supply chain numbers able to satisfy the demand of the OEM more quickly to plunge from 10 to 5 , i.e. by $50 \%$ seems noteworthy. As a result of the rise of indicator from 0.1 to 1.0, avg-time falls from 100 to 56 , i.e. by $44 \%$. The greatest drop ( $24 \%$ ) is noticed in the first step (from 
0.1 to 0.2 ) (Figure. 5). The main conclusion from this part of the simulation experiments is that cooperation with a trading partner with better capacities and an ability to offer greater supply is, apparently, more profitable. It reduces the number of supply chains, too. It is worth to emphasize that the number of supply chains falls together with the concentration of suppliers, which means less work, fewer problems.

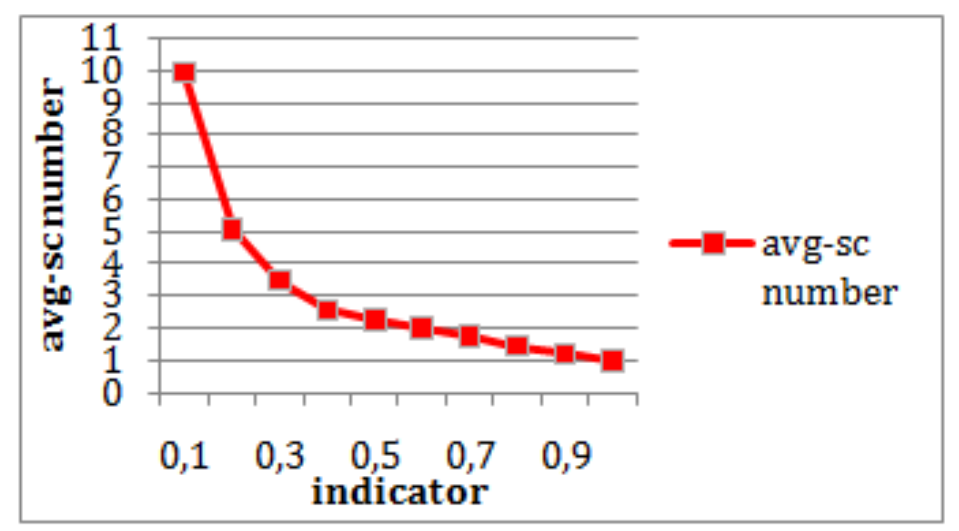

Figure 4. Influence of the factor of supply changeability on the supply chains number in the reverse network

The presented approach allows stimulating different recovery network's configuration. It helps to stabilize the reverse flows of materials from many collection points to single manufacturing/remanufacturing system. The ad hoc testing of recovery network variants is very important in order to match the variable demand and supplies of end-of-life vehicles.

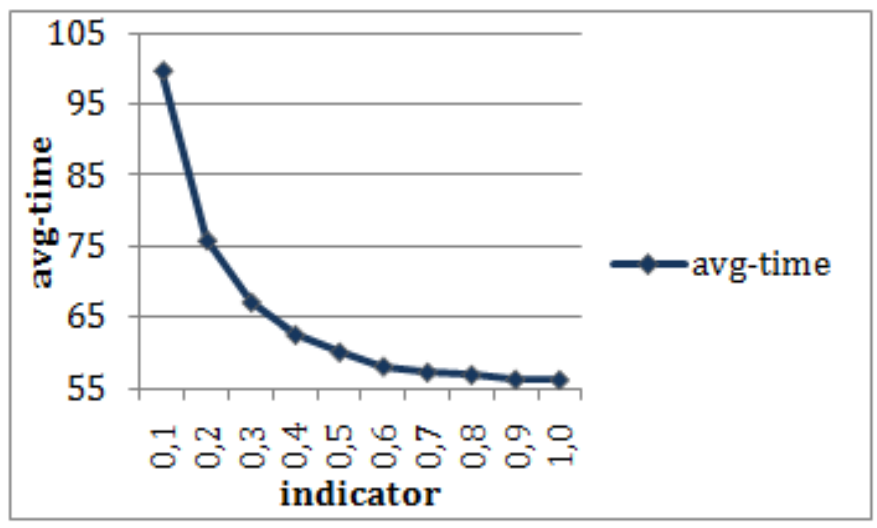

Figure 5. Influence of the factor of supply changeability on the average time of the whole process of the recovery in the network 


\section{Conclusions}

In this paper authors identified the main problems that appear by materials management within remanufacturing system. Application of agent-based technology helps to solve problems that appear very often in recovery network such as:

- distribution of information sources

- heterogonous and incomplete data

- opportunistic approach to network configuration -companies evaluate the benefits of participation in the virtual organization and compare them with benefits of individual performance ( different number of nodes at each triers)

- temporary character of relations

The stability of re-supply in the CLSC was examined by the simulation. The agent technology proved to be suitable for simulation of dynamic network configuration. There is a need for more holistic vision of reverse logistics as part of a closed-loop system. The further research will include in-depth case studies in automotive industry regarding the dynamics of reverse material flows for remanufacturing purpose. In authors opinion there is a need for distributed IT system that allow synchronized material planning and coordination of reverse and forward flows.

\section{References}

ACEA, (2010). Report “Used vehicles 2003-2008", www.acea.be - Accessed 20th December 2010.

Dekker, R., Fleischmann, M., Inderfurth, K., \& Van Wassenhove, L.N. (eds.) (2003). Reverse Logistics: Quantitative Models for Closed-Loop Supply Chains, Springer Verlag, Berlin.

Deo, N., Kowalik, J.S., \& Sysło, M.M. (1983). Discrete Optimization Algorithms with Pascal Programs. Prentice-Hall Inc., Englewood Cliffs.

Directive 2000/53/EC of the European Parliament and of the Council of 18 September 2000 on end-of life vehicles (ELV). 
Directive 2005/64/EC of the European Parliament and of the Council of 26 October 2005 on the type-approval of motor vehicles with regard to their re-usability, recyclability and recoverability and amending Council Directive 70/156/EEC.

EAIR, (2009). European Automobile Industry Report 2009, www.acea.be - Accessed 20th December 2010.

Ferrer, G., \& Whybark, D.C. (2001). Material Planning for a Remanufacturing Facility. Production and Operations Management, 10(2), 112-124. http://dx.doi.org/10.1111/i.1937-5956.2001.tb00073.x

Fleischmann, F., Beullens, P., Bloemhof-Ruwaard, J.M., \& van Wassenhove, L.N. (2001). The Impact of Product Recovery on Logistics Network Design. Production and Operations Management, 10(2), 156-173. http://dx.doi.org/10.1111/j.19375956.2001.tb00076.x

Fleischmann, M., Dekker, R., \& Flapper S.D.P. (2000). A characterization of logistics networks for product recovery, Omega, the international Journal of Management Science, 28, 653-666.

Golinska, P., Fertsch, M., Marx Gomez, J., \& Oleskow, J. (2007). The concept of Closed-loop Supply Chain Integration, Through Agent-based system. Information Technologies in Environmental Engineering, ESE, 203-210, Springer, Berlin Heidelberg.

Guide Jr., V.D.R. (2000). Production planning and control for remanufacturing. J. Oper. Management, 18, 467-483. http://dx.doi.org/10.1016/S0272-6963(00)00034-6

Guide Jr., V.D.R. (2003). Matching Demand and Supply to Maximize Profits from Remanufacturing. Manufacturing and Service operations Management, 5(4), 303316. http://dx.doi.org/10.1287/msom.5.4.303.24883

Guide, Jr., V.D.R., \& Van Wassenhove, L.N. (2003). Business Aspects of ClosedLoop Supply Chains, Carnegie Mellon University Press, Pittsburgh, PA.

Kawa, A. (2009). Simulation of dynamic supply chain configuration based on software agents and graph theory. In: Omatu S. et al (eds.) IWANN 2009 part II. LCNS 5518, 382-389. Springer-Verlag, Berlin, Heidelberg.

Kawa, A., \& Golinska, P. (2010). Supply chain arrangements in recovery network. In: Jedrzejowicz P. et al. (eds.): Agent and Multi-Agent Systems: Technologies 
and Applications, 4th International Conference, AMSTA-10, LNAI 6071, SpringerVerlag, Berlin, Heidelberg.

Kim, H.J., Severengiz, S., Skleros, S., \& Seliger, G. (2008). Economic and environmental assessment of remanufacturing in the automotive industry, 15th CIRP International Conference on Life Cycle Engineering.

Lund, R. (1983). Remanufacturing: United States Experience for Developing Nations. The World Bank, Washington DC.

Seitz, M., Peattie, K. (2004). Meeting the Closed-Loop Challenge: The Case of Remanufacturing. California Management Review, 46(2), 74-89.

SMMT, (2007). 9th Sustainability Report-The UK automotive data 2007, http://www.smmt.co.uk - Accessed 20th December 2010.

Tibben-Lembke, R.S., \& Rogers, D.S. (2002). Differences between forward and reverse logistics in a retail environment. Supply Chain Management: An International Journal, 7(5), 271-282.

Toktay, L.B., Wein, L.M., \& Zenios, S.A. (2000). Inventory management of remanufacturable products, Management Science 46(11): 1412-1426. http://dx.doi.org/10.1287/mnsc.46.11.1412.12082

van der Laan, E.A., Kiesmüller, G., Kuik, R., Vlachos, D., \& Dekker, R. (2003). Stochastic Inventory Control for Product Recovery, in: Dekker, R. et al. (eds.), Reverse Logistics: Quantitative Models for Closed-Loop Supply Chains, Chapter 8, Springer.

Wilensky, U. (2009). NetLogo itself. NetLogo - Center for Connected Learning and Computer-Based Modeling, Northwestern University, Evanston, http://ccl.northwestern. edu/netlogo/.

Journal of Industrial Engineering and Management, 2011 (www.jiem.org)

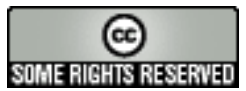

\footnotetext{
Article's contents are provided on a Attribution-Non Commercial 3.0 Creative commons license. Readers are allowed to copy, distribute and communicate article's contents, provided the author's and Journal of Industrial Engineering and Management's names are included. It must not be used for commercial purposes. To see the complete license contents, please visit http://creativecommons.org/licenses/by-nc/3.0/.
} 